\title{
Ethnologies
}

\section{Négocier la transcendance}

\section{Alison Marshall}

Volume 25, numéro 1, 2003

Négocier la transcendance / Negotiating Transcendence

URI : https://id.erudit.org/iderudit/007124ar

DOI : https://doi.org/10.7202/007124ar

Aller au sommaire du numéro

Éditeur(s)

Association Canadienne d'Ethnologie et de Folklore

ISSN

1481-5974 (imprimé)

1708-0401 (numérique)

Découvrir la revue

Citer cet article

Marshall, A. (2003). Négocier la transcendance. Ethnologies, 25(1), 17-28.

https://doi.org/10.7202/007124ar

Ce document est protégé par la loi sur le droit d'auteur. L'utilisation des services d'Érudit (y compris la reproduction) est assujettie à sa politique d'utilisation que vous pouvez consulter en ligne.

https://apropos.erudit.org/fr/usagers/politique-dutilisation/ 


\title{
NÉGOCIER LA TRANSCENDANCE
}

\author{
Alison Marshall \\ Brandon University
}

Ce numéro thématique, "Négocier la transcendance », aborde de front le problème du contrôle de ce phénomène dans les domaines contestés de l'extase religieuse et du chamanisme. En les passant en revue, il va également à l'encontre des présupposés voulant que ces manifestations ne soient que les dérivés de superstitions ou de croyances religieuses populaires qui seraient inférieures aux expériences des religions officielles. En fait, il est fréquent que, de nos jours, de nombreux occidentaux perçoivent ce que l'on appelle les croyances populaires comme étant supérieures aux religions officielles, voire qu'ils renoncent à ces dernières au profit de la spiritualité "New Age » ou du néochamanisme et de leurs voies pour transcender la connaissance (Heelas 1996 ; von Stuckrad 2002). Cette quête d'une connaissance transcendantale a souvent été décrite comme un désir de retour aux origines dans la compréhension spirituelle du monde (Needham 1985 ; Kehoe 1990). Bien que cela puisse s'avérer vrai pour certains, les auteurs de ce numéro, dans leur recherche de nouvelle(s) compréhension(s), ne discutent pas de la connaissance primitive ou primordiale qui serait transmise dans un lieu donné par une divinité précise, mais plutôt de la pluralité des expériences extatiques et des voies par lesquelles celles-ci apportent de nouveaux modes de compréhension(s).

Dans ce numéro, les auteurs recherchent les manières dont les " extatiques », qu'ils soient religieux ou laïcs, ont exprimé et négocié leurs propres expériences de médiums, de chamanes, de danseurs, d'amants ou de guérisseurs. Ces expériences peuvent toutes être dénommées extatiques, en référence à la manière dont une personne devient autre que ce qu'elle est dans la vie de tous les jours, dans des contextes religieux ou laïcs. Lorsque l'on compare les relations 
d'expériences extatiques dans des contextes religieux différents, certains schémas se dessinent. Ainsi, les protestants américains du début du XVIII ${ }^{\mathrm{e}}$ siècle relataient abondamment leurs expériences religieuses au moyen de motifs narratifs décrivant la manière dont ils avaient été remplis de l'Esprit (Taves 1999: 3). À l'occasion de leurs danses, les Boschimans !Kung expliquaient qu'ils essayaient de faire bouillir ce qu'ils appelaient num, ou énergie spirituelle, afin de rendre immanent l'esprit nommé kia (Katz 1982 : 46). Souvent, lorsque le kia était immanent, les gens tremblaient. Les lingji de Taïwan pratiquent la méditation et la danse dans le but de faire bouger l'esprit à l'intérieur d'elles-mêmes (lingdong) et lorsqu'elles ont atteint cet état, elles se mettent aussi à trembler (Marshall 2002). Les protestants américains d'autrefois, les Boschimans !Kung et les lingji de Taïwan utilisent un langage spécifique pour exprimer différentes expériences dans lesquels ils se trouvent remplis de l'esprit, font bouillir l'énergie spirituelle et font bouger l'esprit. Tous ces individus peuvent être définis comme des «extatiques» qui partagent une expérience de l'extase et sont transformés de manière variable par les esprits.

Bien que le terme "extatique "soit commode pour englober les similarités entre religions de traditions différentes, il a fait l'objet de critiques dont nous devons discuter. Roberte Hamayon résume les problèmes que l'on peut rencontrer lorsque l'on utilise des termes tels que « extase » ou « chamane » à propos d'un individu n'étant pas par tradition un chamane sibérien (1998). De nos jours, le terme «chamane» est presque universellement utilisé sans réelle intention de référence à quiconque entre en transe, ou en extase, ou dans un état altéré de la conscience, qu'il soit guérisseur ou possédé. Lorsque j'ai demandé à mes étudiants de troisième année de premier cycle, dans le cours Religions de l'extase dans les traditions mondiales [Ecstatic Religions in World Traditions] de répondre à la question "Qu'est-ce qu'un chamane? », leurs réponses montraient qu'aujourd'hui, au moins en Occident, un chamane peut être défini comme à peu près n'importe quelle personne ayant un rôle spirituel dans n'importe quelle société contemporaine. Les étudiants ont répondu qu'un chamane était le plus souvent de sexe masculin, un prêtre, une liaison avec le monde spirituel, qu'il faisait tomber ou cesser la pluie, un guérisseur, quelqu'un en communication avec les esprits, le guide de sa communauté dans la performance de rituels et de cérémonies, un médium ayant un degré élevé de spiritualité personnelle, quelqu'un pouvant entrer en transe, 
guérir la peste ; il pouvait être bon ou mauvais, être le guide d'une communauté tribale, culturelle ou intellectuelle, un mystique souvent associé aux traditions religieuses de l'Amérique du Nord, de l'Amérique du Sud et de la Sibérie, un magicien et un devin. Il n'y avait pas deux personnes dans la classe à définir un chamane de la même manière. Cependant, lorsque j'ai demandé aux étudiants de définir le domaine d'expérience d'un chamane, tout le monde a répondu qu'un chamane était quelqu'un qui entrait en transe. Alors que, ainsi que le révélaient les réponses à la question "Qu'est-ce qu'un chamane?", n'importe qui pouvait être perçu comme agissant comme un chamane à un moment ou à un autre de sa vie.

Malheureusement, cependant, le terme «chamane » est devenu une dénomination de prédilection pour plusieurs disciplines qui l'utilisent pour identifier ces individus que l'on nommerait ailleurs médiums ou guérisseurs. De plus, l'usage d'une terminologie alternative pour décrire les médiums et les individus agissant comme des chamanes, telle que «acteur extatique» (Paper 1996 ; Marshall 2000), ne va pas sans poser d'autres problèmes dans les cours. En entendant ces mots, les étudiants pensent qu'ils font référence au sexe ou à la drogue qu'on appelle Extasy. Ils sont toujours plus à l'aise avec le terme "chamane ", même si sa définition est comprise de manière très largement englobante. C'est la raison pour laquelle j'utilise souvent la définition du chamane d'Åke Hultkranz, afin d'aider les étudiants à comprendre de quelle manière un chamane expérimente l'extase et agit à l'intérieur d'un contexte religieux et social (1974: 34).

Mais comment désigner ceux qui, sans être des chamanes traditionnels de Sibérie, ont cependant des expériences extatiques ou transcendantes? Devrait-on les appeler néochamanes afin de refléter les influences des croyances New-Age sur leurs pratiques (Hamayon 1998) ? Ou devrait-on y faire référence comme à de modernes chamanes occidentaux, synthétisant les influences chamaniques et "néopaïennes » de citadins américains et européens (von Stuckrad 2002 : 774 ; Høst 2001) ? Si des individus expérimentent une forme de chamanisme dans le cyberespace ou à une rave, devrait-on alors les appeler technochamanes (Rushkoff 1994) ? Certains s'opposent à toute utilisation de termes incluant le mot « chamane », préférant le mot " médium ». Cependant, la signification du mot "médium » lui-même est contestée (Donovan 2000 ; Lewis 1978 ; Rouget 1985). D’autres, dont je fais partie, préfèrent le mot «extatique » pour décrire des 
expériences similaires, sous certains aspects, aux expériences des chamanes (voir Eliade 1951 ; Hultkranz 1974 ; Lewis 1978 ; Paper 1996 ; Marshall 2000). Les auteurs travaillant dans le domaine de l'expérience religieuse doivent éviter l'imposition des énoncés essentialistes de l'impérialisme culturel occidental en abordant ces expériences religieuses (Taussig 1991 ; Hutton 2001). D’un autre côté, ces auteurs doivent pouvoir utiliser les mots extatique, chamane et médium pour procurer un cadre de référence adéquat aux analyses comparatives. Chacun doit soigneusement négocier ces termes en les définissant clairement, tout en sachant finalement que tous les mots ont une histoire.

Mon intérêt, en rassemblant ces articles, provient de mon étude sur l'expérience religieuse taïwanaise et plus particulièrement des expériences d'individus aux dénominations variées, chamanes, néochamanes, médiums, guérisseurs et acteurs rituels. Ces expériences extatiques de nature transcendantale font se rassembler temporairement le corps et l'esprit, le spirituel et le rationnel, le collectif et l'individuel (Sangren 1991) ${ }^{1}$. Afin d'illustrer ces négociations dans le contexte de Taïwan, permettez-moi de raconter une de mes récentes expériences de terrain ${ }^{2}$.

J'étudiais les pratiques religieuses des «voyants de l'esprit » ou lingji à Taïwan ; ils rotaient, chantaient et dansaient pour faire bouger l'esprit en eux. Lors de discussions avec des lingji, majoritairement des femmes, j'ai remarqué qu'elles mentionnaient souvent la manière dont elles établissaient des relations avec différentes divinités, par des moyens autodidactes, en purifiant leurs corps et leurs esprits afin qu'un ling, défini comme l'efficience ou le pouvoir magique (Sangren 1993), d'un esprit vienne les remplir et les inspirer pour créer des danses et des chansons. Cette pratique consistait en une séance statique de méditation assise et en une seconde séance dynamique durant laquelle chacune travaillait avec les autres pour créer des mimes, des danses et des chansons. Mais rien n'était absolument normé dans ces deux étapes de la pratique. Les individus agissaient différemment à chaque étape de la pratique, en fonction de leur niveau de connaissance. Les divinités qui poussaient les participantes à danser étaient rarement les mêmes et les performances, qu'il s'agisse de danse ou de chanson, étaient sans cesse changeantes.

1. Ces expériences diffèrent de celles des mystiques chrétiens qui s'unient à Dieu.

2. Cette recherche a été financée par une bourse du Conseil de Recherche en Sciences Humaines du Canada. 
J'eus l'occasion d'observer une séance qui se déroula de manière totalement impromptue chez un glacier. Cinq lingji m'avaient emmenée manger une glace pour discuter des éléments fondamentaux de la pratique du lingji. J'appréciais grandement cette chance d'en apprendre davantage sur la pratique du lingii et j'espérais que l'une d'entre elles au moins m'expliquerait ce que cela signifiait, être une lingji.

Au lieu de cela, elles m'ont montré ce que signifiait être une lingji en m'enseignant à négocier la transcendance. Lorsque tout le monde eut fini de manger, les assiettes, verres, tasses et soucoupes devinrent les accessoires de la leçon. Tout le monde fit le silence quand Liu, la lingji la plus âgée, praticienne autodidacte depuis plus de dix ans et ayant atteint un niveau élevé, prit soudain un verre rempli d'eau et le vida. Ensuite elle plaça le verre dans une tasse, vide elle aussi. Xiao, une lingji pratiquant depuis trois ans, prit immédiatement une tasse à café vide et la plaça sur une soucoupe. Elle prit ensuite une autre soucoupe et la déposa sur la tasse. Joyce, autre lingji pratiquant depuis trois ans, prit ensuite le verre et la tasse et les plaça au sommet de la pile, soucoupe, tasse et soucoupe. Xiao ajouta de l'eau d'un autre verre à cette structure. Finalement, chacune à table faisait passer l'eau des verres aux tasses en faisant monter une tour d'assiettes, verres, tasses et soucoupes. Ensuite les verres furent déplacés de la pile du bas vers le haut de la table et disposés en forme de croix. Ensuite, les femmes lingji se mirent chacune à son tour à déplacer les soucoupes et les tasses pour transférer l'eau d'un récipient à l'autre.

Plus tard on me dit que le mouvement de l'eau était censé représenter comment s'exerçait le mouvement du qi et du ling d'une personne à l'autre et d'une matière à une autre. Chaque personne offrait sa contribution au mouvement de l'eau (soit ling et qi), et nous avons toutes été unies par notre participation à cet exercice. Lorsque cet exercice fut terminé, un autre commença. Liu réunit son pouce et ses doigts du milieu, se pencha vers chacune à table, puis commença à faire marcher ses doigts sur la surface de la table, s'arrêtant devant la personne se tenant en face d'elle pour permettre à cette personne de continuer cette danse des doigts. Tout au long de cet exercice, chacune était concentrée et sérieuse. Je fus invitée à entrer dans le mouvement quand les doigts de Liu s'arrêtèrent en face de moi, agrippant mes petits doigts et les pressant avec une grande force. Lorsqu'elle m'a pressé les doigts, je me suis rappelée ce qu'elle avait dit au sujet de la décharge électrique dont elle se sentait traversée lorsque l'esprit la poussait à 
agir. Je fis une simple petite danse des doigts, suffisante pour que cet état d'esprit se communique à moi et terminai en indiquant Liu. Cela continua pendant une autre demi-heure (Marshall 2002).

Comment pouvons-nous définir ce que fait la lingji dans ce contexte chinois? Cette pratique n'entre dans aucune des religions chinoises traditionnelles ou officielles: elle n'est ni bouddhiste, ni taoïste, ni confucéenne, ni même New-Age — terme souvent inapproprié pour décrire de nouveaux développements ou mouvements religieux (Melton 2003). Lors des entrevues, les lingji font très attention à se distinguer d'autres personnes qui entrent en transe, comme les médiums (jitong) et les maîtres des rituels taoïstes. Elles ne sont pas non plus des chamanes «traditionnelles» comme en Sibérie ni des néochamanes. Ce que sont les lingii se définit par ce qu'elles font : faire bouger l'esprit (lingdong).

Cette action, faire bouger l'esprit, n'était décrite que très vaguement dans toutes les entrevues par ce terme, lingji. Cela m'a amenée à m'interroger : si les femmes lingji n'utilisent pas de terminologie précise pour ce qu'elles font, cela ne serait-il pas un geste d'impérialisme culturel que de réduire ce qu'elles me disaient pour que cela devienne plus précis?

Ce que je veux exprimer par là est qu'il n'existe peut-être pas de voie précise par laquelle articuler, de manière appropriée, les expériences de personnes telles que les femmes lingji qui deviennent habitées par l'esprit. À la fin, je décidais de m'en remettre aux propres mots des femmes lingji et à mes propres expériences de participante, pour être sûre que leurs propres perceptions et croyances seraient exprimées le plus justement possible (Agar 1980: 104).

Toutes ces femmes lingji se définissaient elles-mêmes au moyen du terme ling — se définissant parfois comme des lingji (voyantes de l'esprit), parfois comme des lingmei (médiums) et d'autres fois encore comme des lingxiu (vouées à l'esprit). Je peux définir ces femmes en tant qu' « extatiques » et «transformées » (en me basant sur ce qu'elles m'ont dit lors des entrevues) en raison du point commun qu'est le ling dans les deux étapes de la pratique. Indépendamment du fait qu'elles aient négocié la transcendance de manière statique par la méditation ou de manière active par la danse (Fischer 1971), elles étaient dans une certaine mesure remplies de ling. Alors que le ling remplissait ces femmes, elles ont commencé à roter et trembler — on dit souvent du ling qu'il 
possède une charge électrique ou magnétique (Nickerson 2001 : 194). Mais la transcendance ne se limitait pas à une personne et au bout du compte cela devint une expérience collective (Sangren 1991). Le ling était contagieux et d'autres, se tenant près de ceux qui étaient en méditation, furent entraînées dans la performance, inspirées par l'esprit, se joignant aux danses déjà en cours, y ajoutant et improvisant.

La pratique du lingji requiert qu'un individu lingji entre dans une sorte de transe dans laquelle il ou elle devient animé par l'esprit, à la fois dans la méditation et dans la danse. Je considère les deux étapes de cette pratique comme des expériences d'extase puisque, durant les deux étapes, l'individu est rempli de ling. Ainsi que le considèrent plusieurs des auteurs de ce numéro, l'individu en extase n'est pas le participant involontaire d'un processus contrôlé par quelque force inconnue. Plus exactement, l'individu en extase négocie son expérience unique, l'utilise comme fin, définissant cette expérience pour lui-même, ou elle-même, en prenant le parti de se vouer à la pureté et à la moralité. Ces négociations déterminent la qualité de la transcendance. Dans un contexte moderne, négocier cette expérience d'extase est un voyage spirituel dans lequel chacun tente de déclencher ce fugitif sens de l'unité avec le divin ou avec l'absolu.

Nous avons demandé aux auteurs de ce numéro de sonder ce sujet, la négociation de la transcendance, et d'interroger en particulier la manière dont se déroulent ces négociations dans des contextes d'extase aussi disparates que la danse, la religion, le théâtre ou l'amour (Turner 1990 : 13). Les approches de ces auteurs varient pour chacun de ces champs, mais dans chacun de ces articles reste constante la volonté de mettre en avant du discours la voix de celui qui expérimente, du protagoniste. Ces «voix» sont souvent les seules sources d'information sur des groupes ou des individus au sujet desquels il n'existe pas de corpus canonique ou académique (Sutcliffe $2000: 6$ ). Bien que la parole du chercheur, du théoricien ou du praticien ait une place dans ces discussions, elle se borne à placer l'autre dans son contexte et ne vise qu'à clarifier et détailler les affirmations déjà prononcées par celui qui expérimente. Bien que ces négociations aient lieu dans des contextes dissemblables, elles démontrent l'importance de la transcendance (ou des transcendances, qui sous-entendent la pluralité des expériences). Ces négociations créent de nouvelle(s) compréhension(s) — sorte de connaissance idéale - que l'on considérait autrefois comme le domaine exclusif du chamane traditionnel et qui, comme l'expérience elle-même, 
est extraordinaire et provient d'un autre monde, que ce monde soit religieux ou simplement « autre».

Les extatiques tels que les chamanes, les médiums, les amoureux, les artistes et les danseurs sont des canaux permettant la connection entre l'état terrestre ordinaire de l'existence et les états extraordinaires. Il nous rappellent l'aptitude humaine au changement et à la transformation. Il nous arrive souvent de regarder ces extatiques à travers le prisme de notre propre situation, de notre expérience de vie, de nos attentes et de nos champs d'étude (Tweed 2002). Les auteurs de ce numéro explorent les différentes voies par lesquelles opère la transformation. Ainsi que l'explique Alice Ormiston, pour Hegel ce canal est l'expérience de l'amour et l'union avec l'absolu. Brenda Cantelo démontre comment, pour la danseuse, c'est le mouvement qui communique sa compréhension d'elle-même à l'animus et sa transformation. Pour Philip Clart et Brigitte Baptandier, qui en discutent dans le contexte de Taïwan et de la Chine du Sud, le canal se trouve entre le dieu et le médium et a pour intermédiaire la morale et l'autodidaxie, respectivement. Souvent les extatiques modèlent l'harmonie, se sacrifiant pour les besoins de leur communauté ou ceux de leur famille de coeur. Dans le travail de Lisa Kuly, nous voyons les chamanes se placer eux-mêmes au centre, puis être renvoyés aux marges une fois le rituel terminé, revenant à l'ordinaire une fois de plus. Tous ces auteurs ont essayé, dans leurs articles, de subjuguer leurs propres négociations et de considérer comment chaque extatique définit son expérience de la transcendance.

L'article de Roberte Hamayon, «Faire des bonds fait-il voler l'âme? De l'acte rituel en Sibérie chamaniste », nous offre un synopsis des différentes manières par lesquelles les universitaires négocient les compréhensions de l'expérience du chamane sibérien. Hamayon, en particulier, nous fait faire un survol et nous donne une bonne définition des termes utilisés dans ce numéro : négociation, transcendance, extase et transe. Cela est suivi de l'exposé que fait l'auteur de l'idée que le rituel sibérien se caractérise par le jeu.

L'article d'Alice Ormiston, "Hegel on Negotiating Transcendence », discute de l'aliénation de la conscience occidentale moderne, séculière, orientée vers un but, qui s'est dissociée de la totalité spirituelle du corps et de l'âme. Ormiston explique ici, avec une grande acuité de détail, l'impact que produit la séparation du religieux et du 
laïc sur l'individu moderne occidental en déspiritualisant notre existence quotidienne. Pour Ormiston, « expérimenter le divin devient ainsi hautement problématique pour les individus modernes ». En développant l'idée d'amour et de vie éthique de Hegel, Ormiston explore la contribution de Hegel aux discussions contemporaines sur la transcendance et l'extase.

Brenda Cantelo, dans «Art as a Way of Knowledge », s'exprime à la fois par la voix de l'universitaire et de la danseuse pour guider le lecteur dans l'exploration d'une danse d'inspiration jungienne. Dans cet écrit, Cantelo négocie ses propres négociations de la transcendance, esquissant des interprétations viscérales, corporelles, spirituelles et intellectuelles de la danse. Pour Cantelo, ces négociations complexes deviennent un processus de compréhension de soi, dans lequel elle prend conscience de l'imagerie jungienne qui culmine en un sens de plénitude. Cantelo écrit : "reconnaître ces images fait partie du processus de compréhension de soi qui, selon Jung, amène de manière ultime la personnalité à un niveau de fonctionnement plus élevé et plus complet».

L'article de Brigitte Baptandier, «Façonner la divinité en soi. À la recherche d'un lieu d'énonciation », explore la manière par laquelle l'identité est mise en forme par les femmes médiums appelées xiangu dans la province du Fujian en Chine. L'auteur examine la médiumnité du point de vue de l'apprentissage, de la destinée, ainsi que la divinité qui les possède. Baptandier décrit ici comment la future xiangu finit par accepter sa destinée karmique et s'embarque pour une mission mystique d'élaboration du soi. Cette mission devient une sorte de but qui l'amène à servir les autres. La mise en forme est une sorte de négociation de la transcendance qui succède à une période d'ascétisme et d'apprentissage menant à la transe et qui est comprise comme une expression métonymique de l'autobiographie.

Dans «Moral Mediums: Spirit-Writing and the Cultural Construction of Chinese Spirit-Mediumship », Philip Clart présente la médiumnité comme un champ culturel contesté de la religion populaire chinoise. À partir de ses propres recherches de terrain, de sources premières comme les livres de morale et d'études de la médiumnité à travers la sphère culturelle chinoise, il démontre l'existence d'interprétations différentes et parfois rivales de la médiumnité. Il décrit en particulier les conceptions ayant cours dans les cultes taïwanais 
d'écriture automatique (les «maisons du phoenix »), où la médiumnité est régie par les lois d'un univers moral. Puisque les dieux sont des forces morales, le médium qui en est possédé doit être une personne à qui ses qualités morales donnent des affinités avec les dieux. Les qualités morales deviennent le présupposé et la base de la transcendance et l'union de la divinité et du médium est ainsi perçue comme se produisant entre deux entités d'essence semblable.

Dans «Locating Transcendence in Japanese Minzoku Geinô : Yamabushi and Miko Kagura », Lisa Kuly fait un exposé historique des minzoku geinô (acteurs populaires) dans la société japonaise, soulignant la manière créative par laquelle des extatiques telles que les femmes chamanes appelées miko ont négocié leurs positions sociales et religieuses. Kuly évoque la montée en puissance sur le plan religieux de ces individus dont le statut liminaire leur permet d'agir en tant que guérisseurs et exorcistes, se déplaçant des marges vers le centre durant un rituel.

Depuis le jeu dans les rituels sibériens jusqu'à la danse dans le Manitoba, la transcendance se négocie partout, souvent dans des endroits inattendus, créant des réseaux de compréhension et d'abondantes réserves de récits personnels en ce qui concerne la portée des expériences extatiques dans les sociétés occidentales modernes. 


\section{References}

Agar, Michael, 1980, The Professional Stranger : An Informal Introduction to Ethnography. New York, Academic Press.

Donovan, James M., 2000, « A Brazilian Challenge to Lewis's Explanation of Cult Mediumship », Journal of Contemporary Religion 15 (3) : 361-377.

Eliade, Mircea, 1964 (1951), Shamanism : Archaic Techniques of Ecstasy. Princeton (NJ), Princeton University Press.

Fischer, Roland, 1971, "A Cartography of the Ecstatic and Meditative States », Science 174 (26 November) : 897-904.

Hamayon, Roberte, 1998, «Ecstasy or the West-dreamt Shaman », dans Helmut Wautischer (dir.), Tribal Epistemologies. Aldershot, Ashgate (Averbury Series in Philosophy) : 175-187.

Heelas, Paul, 1996, The New Age Movement: The Celebration of the Self and the Sacralization of Modernity. Cambridge MA, Blackwell Publishers.

Høst, Annette, 2001, "What's in a Name? Neo-Shamanism, Core Shamanism, Urban Shamanism, Modern Shamanism, Modern Shamanism or What? ", Spirit Talk 14: 3-6.

Hultkranz, Åke, 1974, «A definition of Shamanism », Temenos 9 : 34. Hutton, Ronald, 2001, Shamans, Siberian Spirituality and the Western Imagination. London, New York University Press.

Katz, Richard, 1982, Boiling Energy : Community Healing among the Kalahari Kung. Cambridge MA, Harvard University Press.

Kehoe, Alice B., 1990, «Primal Gaia : Primitivists and Plastic Medicine Men », dans James A. Clifton (dir.), The Invented Indian. New Brunswick, Transaction : 193-209.

Lewis, Ioan M., 1978 (1971), Ecstatic Religion : an Anthropological Study of Spirit Possession and Shamanism. Great Britain, Hazell.

Marshall, Alison R., 2002, "When the spirit moves on Taiwan. New Religious Movements section ». Paper presented at the American Academy of Religion, National Meeting, Toronto, November 23, 2002 .

, 2000, From the Chinese religious ecstatic to the Taiwanese Theatre of Ecstasy: A Study of the Wu. (Ph.D. Dissertation, University of Toronto).

Melton, J. Gordon, 2003, «An Introduction to New Religions », dans James R. Lewis (dir.), The Oxford Handbook for New Religious Movements. New York, Oxford University Press. 
Needham, Rodney, 1985, Exemplars. Berkeley, University of California Press.

Nickerson, Peter, 2001, "A Poetics and Politics of Possession : Taiwanese Spirit, Medium Cults and Autonomous Popular Cultural Space », Positions 9 (1) : 187-217.

Paper, Jordan, 1996, « Mediums and Modernity : the Institutionalization of Ecstatic Religious Functionaries in Taiwan », Journal of Chinese Religions 24 : 105-129.

Rouget, Gilbert, 1985 (1980), Music and Trance: A Theory of the Relations between Music and Possession. Chicago, University of Chicago Press.

Rushkoff, Douglas, 1994, Cyberia: Life in the trenches of hyperspace. New York, Harper Collins.

Sangren, Stephen, 1991, "Dialectics of Alienation : Individuals and Collectivities in Chinese Religion ", Man 26: 67-86.

— 1993, "Power and Transcendence in the Ma Tsu Pilgrimages of Taiwan », American Ethnologist 20 : 264-282.

Sutcliffe, Steven and Marion Bowman, 2000, Beyond New Age: Exploring Alternative Spirituality. Edinburgh, Edinburgh University Press.

Taussig, Michael, 1991, Shamanism, Colonialism and the Wild man : A Study in Terror and Healing. Chicago, University of Chicago Press.

Taves, Ann, 1999, Fits, Trances, and Visions : Experiencing Religion and Explaining Experience from Wesley to James. Princeton, Princeton University Press.

Turner, Victor, 1990, "Are there universals of performance in myth, ritual, and drama? ", dans Richard Schechner and Willa Appel (dir.), By Means of Performance: Intercultural studies of theatre and ritual. Cambridge, Cambridge University Press : 8-18.

Tweed, Thomas, 2002, «On Moving Across: Translocative Religion and the Interpreters Position », Journal of the American Academy of Religion 70 (2) : 253.

von Stuckrad, Kocku, 2002, "Reenchanting Modern Western Shamanism and Nineteenth Century Thought ", Journal of the American Academy of Religion 70 (4) : 771-799. 